\title{
Afilamento escleral em paciente com rinosporidiose conjuntival
}

\section{Scleral thinning in a patient with conjunctival rhinosporidiosis}

Patrick Frensel de Moraes Tzelikis', Herval Cavalcante Martins², Graziella Vilela ${ }^{3}$, Leonardo Akaishi ${ }^{4}$

\section{Resumo}

Os autores descrevem um caso de rinosporidiose conjuntival acompanhado de afilamento escleral, tratado cirurgicamente com enxerto escleral. São analisadas semelhanças e diferenças com outros casos descritos na literatura.

Descritores: Rinosporidiose; Conjuntivite; Granuloma; Neoplasias da conjuntiva; Esclera/patologia; Relatos de casos [Tipo de publicação]

\footnotetext{
'Pós-graduando pela Faculdade de Medicina da Universidade Federal de Minas Gerais - UFMG - Belo Horizonte (MG), Brasil; Assistente do Serviço de Córnea e Catarata do Hospital Oftalmológico de Brasília - HOB - Brasília (DF), Brasil;

${ }^{2}$ Residente em Oftalmologia do Hospital das Clínicas da Faculdade de Medicina da Universidade de São Paulo - USP - São Paulo (SP), Brasil;

${ }^{3}$ Residente em Oftalmologia do Hospital de Base do Distrito Federal - HBDF - Brasília - (DF), Brasil;

${ }^{4}$ Mestre, Médico Chefe do Serviço de Córnea e Catarata do Hospital Oftalmológico de Brasília - HOB - Brasília (DF), Brasil.
}

Trabalho realizado no Serviço de Córnea, Catarata e Doenças Externas do Hospital Oftalmológico de Brasília - HOB. Brasília - DF. 


\section{INTRODUÇÃO}

A rinosporidiose é uma infecção granulomatosa na qual o agente etiológico é o Rhinosporidium seeberi, que acomete primariamente as mucosas nasais ou oculares, levando ao aparecimento de reação tecidual crônica, caracterizada pela formação de lesões polipóides e vascularizadas. A mucosa nasal é o sítio de infecção mais freqüente, seguido da conjuntiva bulbar ${ }^{(1-2)}$.

$\mathrm{O}$ agente foi descrito na literatura, pela primeira vez, em 1900, por Guillermo Seeber, após a obtenção de material da cavidade nasal de um paciente de 19 anos, trabalhador rural $^{(3)}$. O primeiro caso com envolvimento ocular foi relatado por Ingram, em 1910 $0^{(4)}$. Desde então, diferentes relatos vêm sendo ocasionalmente apresentados em todo o mundo, principalmente nas regiões da Índia e Sirilanka, onde a rinosporidiose é endêmica ${ }^{(5)}$.

O objetivo deste trabalho é relatar, na literatura nacional, o primeiro caso de rinosporidiose ocular que se apresentou na forma de lesão polipóide na conjuntiva bulbar, associada a afilamento escleral.

\section{Relato De CAso}

E.A.S, 12 anos, masculino, leucodérmico, estudante, natural de Boa Vista - Roraima, residente em Brasília - DF. Procurou o Hospital Oftalmológico de Brasília (HOB) queixando-se do aparecimento de uma pequena lesão nodular na região temporal do olho esquerdo $(\mathrm{OE})$, há 8 meses, com crescimento lento e progressivo. Referia leve desconforto ocular. Negava dor, prurido ou baixa visual no olho acometido. Não estava em uso de nenhuma medicação.

O paciente relatava leve trauma ocular contuso, com vegetal, no OE, algumas semanas antes do aparecimento da lesão. Negava cirurgia ocular prévia, assim como doenças sistêmicas.

Ao exame inicial, o paciente apresentava acuidade visual não corrigida normal em ambos os olhos (AO). À biomicroscopia, observava-se pequena lesão conjuntival polipóide, pedunculada, móvel, de consistência macia e coloração avermelhada, na região temporal do OE. Na região subjacente da esclera, observava-se área de adelgaçamento, de $6,0 \times 6,0 \mathrm{~mm}$, através da qual se percebia a coloração azul-acastanhada da úvea. As demais estruturas oculares do $\mathrm{OE}$ não apresentavam qualquer alteração aparente, assim como eram normais todas as estruturas do OD. A pressão intra-ocular registrada foi de 12 e $10 \mathrm{mmHg}$ no OD e OE, respectivamente, às 9:20 horas (Figura 1).
Para esclarecimento diagnóstico, o paciente foi submetido à biópsia excisional da lesão pedunculada, seguida de enxerto escleral na área de afilamento, com o objetivo de restaurar a integridade da parede ocular e evitar complicações futuras. O exame histopatológico (corado por hematoxilina-eosina, PAS e tricrômio de Gomori) evidenciou hiperplasia do epitélio escamoso da conjuntiva e acentuado infiltrado inflamatório crônico, constituído predominantemente por linfócitos, associados a histiócitos, plasmócitos e neutrófilos. Em meio ao infiltrado, observava-se a presença de inúmeros esporângios de Rhinosporidium seeberi, representados por estruturas císticas, eosinofílicas, de duplo contorno e paredes espessas. No pós-operatório, o paciente foi tratado com pomada de antibiótico local e evoluiu sem complicações e sem sinais de recidiva, após 12 meses de acompanhamento.

\section{Discussão}

As infecções oculares fúngicas vêm sendo cada vez mais reconhecidas como uma importante causa de morbidade e cegueira ${ }^{()}$. Os fungos são agentes oportunistas no olho, uma vez que raramente infectam olhos sadios ou que apresentem sua estrutura íntegra. No entanto, mesmo um leve trauma na superfície ocular pode comprometer sua integridade e levar ao aparecimento de um quadro de infecção fúngica ${ }^{(7)}$.

Apesar de identificado desde 1900, Rhinosporidium seeberi ainda apresenta sua patogênese bastante incerta. Em virtude de não se conhecerem seus estágios sexuais, sua classificação taxonômica ainda é bastante discutível. O R. seeberi já foi considerado como um protozoário, e atualmente é classificado como um organismo fúngico anômalo, pertencente à classe Mesomycetozoea, juntamente com outros 10 parasitas e micróbios saprófitas ${ }^{(8)}$.

A conjuntivite por Rhinosporidium seeberi parece ser endêmica em lugares como a Índia e o Sirilanca, onde a prevalência desta doença é estimada em 470 casos por 100.000 habitantes $^{(5-7)}$. No entanto, nos últimos anos, um número significativo de casos foi relatado em outros países, como Estados Unidos, Zaire, Kuwait e Sérvia ${ }^{(6)}$. A maioria dos casos relatados de conjuntivite por rinosporidiose ocorreu em áreas de clima quente e seco. Apesar das evidências epidemiológicas apresentadas em vários trabalhos sugerirem o habitat natural do R. seeberi como sendo locais que apresentam água contaminada, como poços, rios ou lagos, não foi possível sua confirmação através do exame dessas águas ${ }^{(8)}$. Crianças e adultos jovens residentes em áreas rurais continuam a ser os mais 

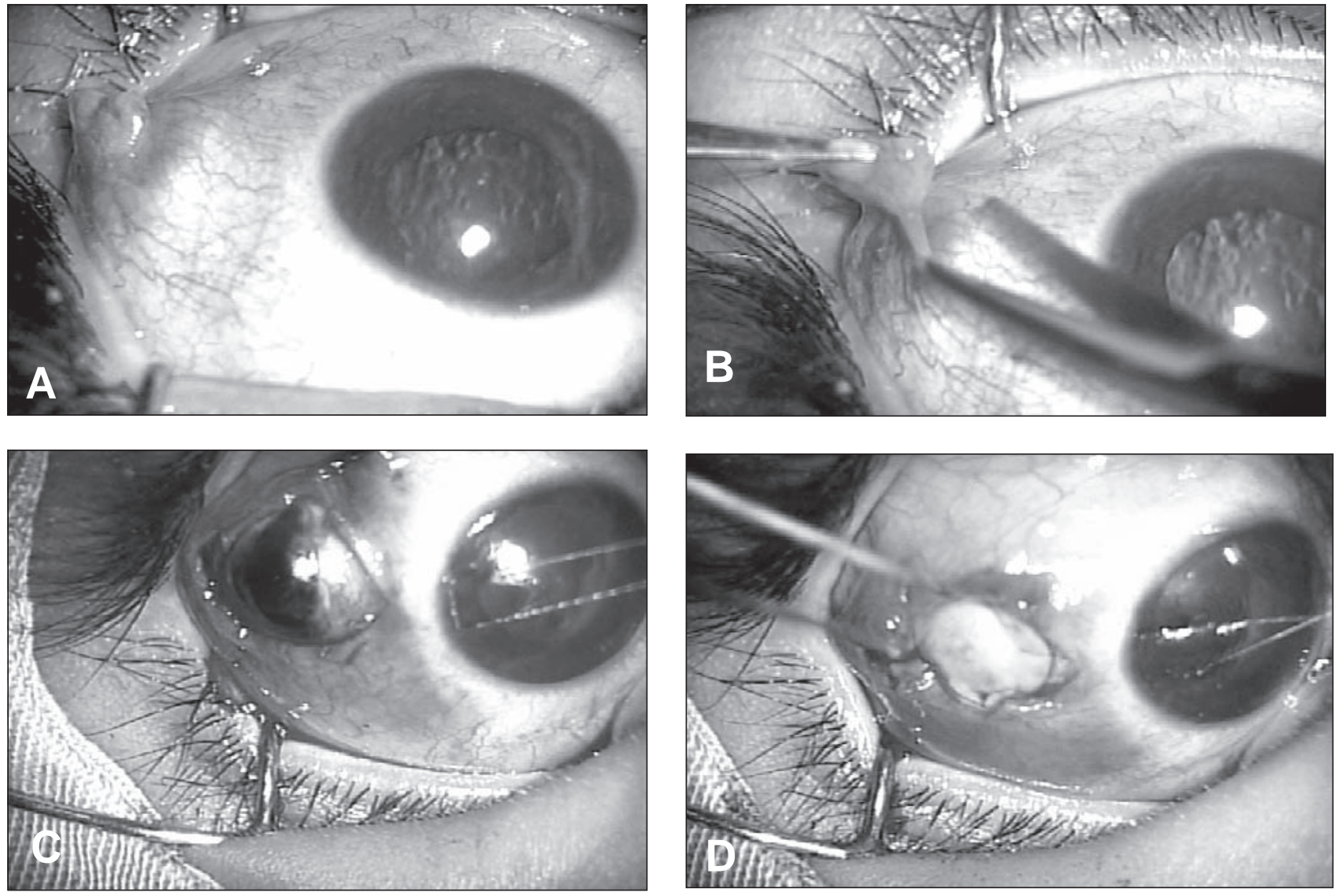

Figura 1: A - Lesão conjuntival polipóide, móvel, vascularizada, avermelhada, sobre área de afilamento escleral na região temporal do olho esquerdo; B - Exérese de lesão conjuntival para exame histopatógico; C - Exposição da área de adelgaçamento escleral; D - Colocação de enxerto escleral sobre a região de afilamento

acometidos por esse agente, sendo que a maioria dos estudos apresenta uma prevalência do sexo masculino em relação ao feminino, numa relação de $4: 1^{(6-7)}$.

$\mathrm{O}$ acometimento de estruturas oculares responde por $15 \%$ de todos os casos de infecção por R. seeberi ${ }^{(1)}$. As lesões oculares são geralmente unilaterais e apresentam-se na forma de uma estrutura pedunculada ou séssil, móvel, com aspecto granular, coloração rosada ou avermelhada, e geralmente aderida ao fórnice ou conjuntiva tarsal ${ }^{(9-1)}$. O envolvimento de outras estruturas oculares, como carúncula, limbo, margem palpebral, vias lacrimais e esclera já foi relatado, porém com menor freqüência ${ }^{(6-7)}$.

A presença de envolvimento escleral pelo $\mathrm{R}$. seeberi é bastante rara, tendo sido descrita na literatura mundial em apenas quatro trabalhos ${ }^{(10-13)}$. O diagnóstico clínico deste tipo de escleromalácia é baseado nos achados conjuntivais concomitantes, porém a confirmação diagnóstica só é possível através do exame histopatológico, no qual se podem identificar os esporângios (lesões de aspecto cístico) em diferentes estágios de maturação e degeneração, circundados por um infiltrado inflamatório crônico, predominantemente constituído por linfócitos, plasmócitos, histiócitos e neutrófilos ${ }^{(1)}$. O paciente em questão apresentava, além do afilamento escleral, uma lesão polipóide na conjuntiva suprajacente. Em todos os outros casos descritos na literatura, não foi observada nenhuma lesão polipóide concomitante, mas apenas uma discreta congestão conjuntival com pontos granulares branco$\operatorname{acinzentados}^{(10,12)}$. A exclusão de outras causas de "melting" escleral de origem não infecciosa deve ser sempre realizada. Escleromalácia perfurante é um importante diagnóstico diferencial, sendo esta mais freqüente em pacientes idosos, com história de artrite reumatóide e com acometimento bilateral ${ }^{(10,12)}$

Entre as opções terapêuticas, a única considerada efetiva é a cirúrgica. As recidivas são raras e decorrem da excisão incompleta da lesão. $\mathrm{O}$ uso de cauterização ou crioterapia na base da lesão parece contribuir para redução das recorrências da doença, hipótese ainda não confirmada $^{(1)}$. O tratamento clínico através de medicações 
tópicas e sistêmicas vem sendo proposto por alguns autores. No entanto, o uso destas drogas não apresentou nenhuma evidência que comprovasse sua real eficácia ${ }^{(1-2,6)}$.

Em conclusão, é descrito o primeiro caso na literatura nacional de rinosporidiose ocular que se apresentou na forma de uma lesão conjuntival associada com afilamento escleral. A rinosporidiose conjuntival deve estar sempre no diagnóstico diferencial de qualquer lesão conjuntival acompanhada de adelgaçamento escleral, em pacientes jovens e sadios.

\section{Abstract}

The authors report a case of conjunctival rhinosporidiosis associated with scleral thinning treated surgically with scleral graft. The similarities and differences with other reported cases in the literature are analyzed.

Keywords: Rhinosporidiosis; Conjunctivitis; Granuloma; Conjunctival neoplasms; Sclera/pathology; Case reports [Publication type]

\section{REFERÊNCIAS}

1. Reidy JJ, Sudesh S, Klafter AB, Olívia C. Infection of the conjunctiva by Rhinosporidium seeberi. Surv Ophthalmol. 1997; 41(5): 409-13. Review.
2. Rippon JW. Medical mycology: the pathogenic fungi and the pathogenic actinomycetes. 3rd ed. Philadelphia: Sauders; 1988. p. $32-72$.

3. Seeber GR. Un nuevo esporozoario parásito del hombre. Dos casos encontrados en pólipos nasales (thesis). Buenos Aires: Universidad Nacional de Buenos Aires; 1900. p. 1-62.

4. Ingram AC, Cantab BC. Rhinosporidium kinealyi in unusual situations. Lancet. 1910; 2: 72.

5. Mukherhee PK. Rhinosporidiosis (oculosporidiosis). In: Fraunfelder FT, Roy FH, editors. Current ocular therapy. 4th ed. Philadelphia, PA: WB Sauders; 1995. p. 77-9.

6. Thomas PA. Current perspectives on ophthalmic mycoses. Clin Microbiol Rev. 2003; 16(4): 730-97.

7. Moses JS, Balachandran C, Sandhanam S, Ratnasamy N, Thanappan S, Rajaswar J, Moses D. Ocular rhinosporidiosis in Tamil Nadu, India. Mycopathologia. 1990; 111(1): 5-8.

8. Arseculeratne SN. Rhinosporidiosis: what is the cause? Curr Opin Infect Dis. 2005; 18(2): 113-8. Review.

9. Boni ES, Saliba ZM, Sessino DB, Miranda JNR, Boni M Rinosporidiose da conjuntiva: relato de caso. Arq Bras Oftalmol. 2002; 65(1): 103-5.

10. De Doncker RM, de Keizer RJ, Oosterhuis JA, Maes A. Scleral melting in a patient with conjunctival rhinosporidiosis. Br J Ophthalmol. 1990; 74(10): 635-7.

11. Kuriakose ET. Oculosporidiosis: rhinosporidiosis of the eye. Br J Ophthamol. 1963; 47: 346-9.

12. Lamba PA, Shukla KN, Ganapathy M. Rhinosporidium granuloma of the conjunctiva with scleral ectasia. Br J Ophthalmol. 1970; 54(8): 565-8.

13. Castelino AM, Rao SK, Biswas J, Gopal L, Madhavan HN, Kumar SK. Conjunctival rhinosporidiosis associated with scleral melting and staphyloma formation: diagnosis and management. Cornea. 2000; 19(1): 30-3. 